\title{
On Certain Conditions of Multivariate Power Series Distributions
}

\author{
Sadoon Abdullah Ibrahim Al-Obaidy ${ }^{1}$, Faisal Ababneh ${ }^{2}$, \\ S. Alwadi ${ }^{3}$, Feras M. Al- Faqih ${ }^{4}$ \\ 1,2,3,4 Department of Mathematics, Al-Hussein Bin Talal University, \\ Ma'an - Jordan \\ sadoon58@yahoo.com \\ , Fmababneh@gmail.com \\ sadam_alwadi@yahoo.co.uk \\ oxfer@yahoo.com
}

\begin{abstract}
During the last decades, no researches have conducted in order to prove some properties of the of the multivariate power series distribution, as results of the present study proved that any multivariate power series distribution is determined uniquely from the mean -function of any marginal random variable. Furthermore these results indicated also that any given function satisfying certain conditions construct a random vector with multivariate power series distribution which has a mean of the marginal random variable. A useful technique can be applied in model building when we have information about the mean-function.
\end{abstract}

Key Words: Multivariate Power Series Distributions, Defining Function, Maclaurin Expansion, Multivariate Logarithmic Distribution, Truncated Power Series Distributions.

\section{Introduction}

Many authors deal with multivariate power series distributions and its theories. Korwar (1975), Dehiya and Korwar (1977), Katri (1978a, 1978b), Papageorgiou(1985), Kyriakoussis and Papageorgiou(1989) studied the generalization of characterization by conditional distributions and regression 
Sadoon Abdullah Ibrahim Al-Obaidy et al.

function. Ghosh ,et.al., (1977) considered on his paper a characterization of positive and negative multinomial distributions and some properties of multivariate power series distributions. Rao and Janardan (1982) discussed a general approach to findings the moments of two classes of multivariate discrete distribution. Gupta and Das (2008) derived anew distribution called the quasi multivariate logarithmic series distribution (QMLSD) of order $\mathrm{k}$ from the multivariate able series distribution(MASDs) of order k. Simic (2009) calculated the moments of distributions as inflated parameter distribution has been discussed by Momeni (2011). Mahmoudi and Jafari (2012) obtained a new class of distribution contains several lifetime model by compound generalized exponential and power series distributions. Silva, et.al., (2013) introduced a new class of distributions which obtained by compounding the extended Weibull and power series distributions .However, the mentioned researchers have not discussed some properties of multivariate power series, such that the unique and the mean of marginal random variable. Therefore, the present study is an attempt to prove the followings:

Firstly: Any multivariate power series distribution is determined uniquely from the mean-function of any marginal random variable.

Secondly: Any given function satisfying certain conditions constructs a random vector with multivariate power series distribution which has a mean of the marginal random variable. This paper is organized as follows, the first section is the introduction the second section is the methodology then the third section is an empirical example then the forth section is an open problem, then finally is the conclusion.

\section{Proposed Method}

\section{Methodology}

Let $f\left(\theta_{1}, \theta_{2}, \ldots, \theta_{k}\right)=\sum_{x_{1} x_{2} \ldots x_{k}} a_{x_{1} x_{2} \ldots x_{k}} \theta_{1}^{x_{1}} \theta_{\theta_{2}}^{x_{2}} \ldots \theta_{k}^{x}, \quad a_{x_{1} x_{2} \ldots x_{k}} \geq 0$, be a convergent series for $\left(\theta_{1}, \theta_{2}, \ldots, \theta_{k}\right) \in \pi_{i=1}^{k}\left[\mathbf{0}, \mathbf{r}_{i}\right], r_{i}$ is a real number or infinity, the intervals $\left[\mathbf{0}, \boldsymbol{r}_{i}\right]$ are non-intersecting or overlapping, and $\boldsymbol{x}_{\boldsymbol{i}}=\boldsymbol{n}_{\boldsymbol{i}}, \boldsymbol{n}_{\boldsymbol{i}}+\mathbf{1 ,} \boldsymbol{n}_{\boldsymbol{i}}+\mathbf{2}, \ldots$ where $\boldsymbol{n}_{\boldsymbol{i}}, \mathbf{i}=\mathbf{1}, \mathbf{2}, \ldots, \mathbf{k}$ is non-negative integer which maybe the same for all i or different for each I, for more details and discussion refer to (Katri, 1959; Ghosh, et. al., 1977).

$$
\text { Let } p\left(X_{1}=x_{1}, X_{2}=x_{2}, \ldots, X_{k}=x_{k}\right)=\mathbf{a}_{x_{1} x_{2} \ldots x_{\mathrm{k}}} \theta_{1}^{x_{1}} \theta_{2}^{x_{2}} \ldots \theta_{\mathrm{k}}^{x_{\mathrm{k}}} / f\left(\theta_{1}, \theta_{2}, \ldots, \theta_{\mathrm{k}}\right) \text {. }
$$

It is easy to see that:

$$
p\left(X_{1}=x_{1}, X_{2}=x_{2}, \ldots, X_{k}=x_{k}\right) \text { is defined if } \theta_{i} \in\left(0, r_{i}\right), \mathrm{i}=\mathbf{1}, \mathbf{2}, \ldots, \mathrm{k} . \text { Also }
$$




$$
\begin{aligned}
& \lim _{\theta_{i} \rightarrow 0^{+}} p\left(X_{1}=x_{1}, X_{2}=x_{2}, \ldots, X_{k}=x_{k}\right)=\lim _{\theta_{i} \rightarrow 0^{+}} \frac{a_{x_{1} x_{2} \ldots x_{k}} \theta_{1}^{x_{1}} \theta_{2}^{x_{2} \ldots \theta_{k}^{x} k}}{f\left(\theta_{1}, \theta_{2}, \ldots, \theta_{k}\right)} \\
& =\lim _{\theta_{i} \rightarrow 0^{+}} \frac{a_{x_{1} x_{2} \ldots x_{k}} \theta_{1}^{x_{1}} \theta_{2}^{x_{2} \ldots \theta_{k} k}}{\sum_{x_{i}} \psi_{x_{i}} \theta_{i}^{x_{i}}} .
\end{aligned}
$$

Where $\psi_{x_{i}}=\sum_{x_{i} \ldots x_{i-1} x_{i+1} \ldots x_{k}} a_{x_{1} \ldots x_{i-1} x_{i+1} \ldots x_{k}} \theta_{1}^{x_{1}} \ldots \theta_{i-1}^{x_{i}-1} \theta_{i+1}^{x_{i}+1} \ldots \theta_{k}^{x_{k}}$, is independent of $\theta_{i} \cdot$

Therefore,

$$
\begin{aligned}
\lim _{\theta_{i} \rightarrow 0^{+}} p\left(X_{1}=\right. & \left.x_{1}, X_{2}=x_{2}, \ldots, X_{k}=x_{k}\right)=\lim _{\theta_{i} \rightarrow 0^{+}} \frac{a_{x_{1} x_{2} \ldots x_{k}} \theta_{1}^{x_{1}} \theta_{2}^{x_{2}} \ldots \theta_{i}^{x_{i}} \ldots \theta_{\mathrm{k}}^{x_{\mathrm{k}}}}{\psi_{n_{i}} \theta_{i}^{n_{i}}+\psi_{n_{i}+i} \theta_{i}^{n_{i}+1}+\ldots} \\
& =\left\{\begin{array}{ll}
1 \text { if } x_{i}=n_{i}, \mathrm{i}=1,2, \ldots, \mathrm{k} \\
0 \text { if } x_{i} \neq \mathrm{n}_{i}
\end{array}\right\}
\end{aligned}
$$

Using this limiting value we see that $\boldsymbol{P}\left(\boldsymbol{X}_{1}=\boldsymbol{x}_{1}, \boldsymbol{X}_{2}=\boldsymbol{x}_{2}, \ldots, \boldsymbol{X}_{\boldsymbol{k}}=\boldsymbol{x}_{k}\right)$ is defined for all $x_{i}$ and $\theta_{i} \in\left(\mathbf{0}, r_{i}\right), \mathbf{i}=\mathbf{1}, 2, \ldots, \mathbf{k}$. the above conditions insure that: $P\left(X_{1}=x_{1}, X_{2}=x_{2}, \ldots, X_{k}=x_{k}\right) \geq 0$, for all $x_{i}$ and $\theta_{i} \in\left(0, r_{i}\right)$. Also,

$$
\begin{aligned}
& \sum_{x_{1} x_{2} \ldots x_{k}} P\left(X_{1}=x_{1}, X_{2}=x_{2}, \ldots, X_{k}=x_{k}\right)= \\
& \frac{1}{f\left(\theta_{1}, \theta_{2}, \ldots, \theta_{k}\right)} \sum_{x_{1} x_{2} \ldots x_{k}} a_{x_{1} x_{2} \ldots x_{k}} \theta_{1}^{x_{1}} \theta_{2}^{x_{2}} \ldots \theta_{k}^{x_{k}}=1
\end{aligned}
$$

Because of these properties we say that $\left(\boldsymbol{X}_{1}, \boldsymbol{X}_{2}, \ldots, \boldsymbol{X}_{k}\right)$ is a random vector with multivariate power series distribution in $\mathrm{k}$-parameters.

The function $f\left(\theta_{1}, \theta_{2}, \ldots, \theta_{k}\right)$ called the defining function of the distribution of the random vector $\left(X_{1}, X_{2}, \ldots, X_{k}\right)$.

The mean function of the marginal random variable $X_{i}$ is : 
Sadoon Abdullah Ibrahim Al-Obaidy et al.

$$
\begin{aligned}
\mu_{i}\left(\theta_{1}, \theta_{2}, \ldots, \theta_{k}\right) & =\mathrm{EX}_{i} \\
& =\sum_{x_{1} x_{2} \ldots x_{k}} x_{i} P\left(X_{1}=x_{1}, X_{2}=x_{2}, \ldots, X_{k}=x_{k}\right) \\
& =\frac{1}{f\left(\theta_{1}, \theta_{2}, \ldots, \theta_{k}\right)} \sum_{x_{1} x_{2} \ldots x_{k}} x_{i} a_{x_{1} x_{2} \ldots x_{k}} \theta_{1}^{x_{1}} \theta_{2}^{x_{2}} \ldots \theta_{i}^{x_{i}} \ldots \theta_{k}^{x_{k}} \\
& =\frac{\theta_{i}}{f\left(\theta_{1}, \theta_{2}, \ldots, \theta_{k}\right)} \sum_{x_{1} x_{2} \ldots x_{k}} x_{i} a_{x_{1} x_{2} \ldots x_{k}} \theta_{1}^{x_{1}} \theta_{2}^{x_{2}} \ldots \theta_{i}^{x_{i}-1} \theta_{i}^{x_{i}+1} \ldots \theta_{k}^{x_{k}} \\
& =\theta_{i} \frac{\frac{\partial}{\partial \theta_{i}} f\left(\theta_{1}, \theta_{2}, \ldots, \theta_{k}\right)}{f\left(\theta_{1}, \theta_{2}, \ldots, \theta_{k}\right)} \\
& =\theta_{i} \frac{\partial}{\partial \theta_{i}} \ln f\left(\theta_{1}, \theta_{2}, \ldots, \theta_{k}\right)
\end{aligned}
$$

It is clear that $\mu_{i}\left(\theta_{1}, \theta_{2}, \ldots, \theta_{k}\right)$ and $\mu_{i}\left(\theta_{1}, \theta_{2}, \ldots, \theta_{k}\right) / \theta_{i}$ are non-negative and continuous functions for all $\theta_{i} \in\left(\mathbf{0}, \boldsymbol{r}_{i}\right)$.

In fact the two functions are differentiable for all $\boldsymbol{\theta}_{i} \in\left(\mathbf{0}, \boldsymbol{r}_{i}\right)$. Finally,

$$
\begin{aligned}
& \lim _{\theta_{i} \rightarrow 0^{+}} \mu_{i}\left(\theta_{1}, \theta_{2}, \ldots, \theta_{k}\right) \\
= & \lim _{\theta_{i} \rightarrow 0^{+}} \sum_{x_{1} x_{2} \ldots x_{k}} x_{i} P\left(X_{1}=x_{1}, X_{2}=x_{2}, \ldots, X_{k}=x_{k}\right) \\
= & \lim _{\theta_{i} \rightarrow 0^{+}} \frac{\sum_{x_{1} x_{2} \ldots x_{k}} x_{i} a_{x_{1} x_{2} \ldots x_{k}} \theta_{1}^{x_{1}} \theta_{2}^{x_{2}} \ldots \theta_{i}^{x_{i}} \ldots \theta_{k}^{x_{k}}}{\sum_{\mathrm{X}_{i}} \psi_{x_{i}} \theta_{i}^{x_{i}}} \\
= & \lim _{\theta_{i} \rightarrow 0^{+}} \frac{\sum_{x_{i}} x_{i} \psi_{x_{i}} \theta_{i}^{x_{i}}}{\sum_{x_{i}} \psi_{x_{i}} \theta_{i}^{x_{i}}}
\end{aligned}
$$




$$
\begin{aligned}
& =\lim _{\theta_{i} \rightarrow 0^{+}} \frac{n_{i} \psi_{n_{i}} \theta_{i}^{n_{i}}+\left(n_{i}+1\right) \psi_{n_{i}+1} \theta_{i}^{n_{i}+1}+\left(n_{i}+2\right) \psi_{n_{i}+2} \theta_{i}^{n_{i}+2}+\ldots}{\psi_{n_{i}} \theta_{i}^{n_{i}}+\psi_{n_{i}+1} \theta_{i}^{n_{i}+1}+\psi_{n_{i}+2} \theta_{i}^{n_{i}+2}+\ldots} \\
& =\lim _{\theta_{i} \rightarrow 0^{+}} \frac{n_{i}\left(\psi_{n_{i}} \theta_{i}^{n_{i}}+\psi_{n_{i}+1} \theta_{i}^{n_{i}+1}+\ldots\right)+\left(\psi_{n_{i}+1} \theta_{i}^{n_{i}+1}+2 \psi_{n_{i}+2} \theta_{i}^{n_{i}+2}+\ldots\right)}{\psi_{n_{i}} \theta_{i}^{n_{i}}+\psi_{n_{i}+1} \theta_{i}^{n_{i}+1}+\psi_{n_{i}+2} \theta_{i}^{n_{i}+2}+\ldots} \\
& =\lim _{\theta_{i} \rightarrow 0^{+}}\left\{n_{i}+\frac{\theta_{i}^{n_{i}+1}\left(\psi_{n_{i}+1}+2 \psi_{n_{i}+2} \theta_{i}+\ldots\right)}{\theta_{i}^{n_{i}}\left(\psi_{n_{i}}+\psi_{n_{i}+1} \theta_{i}+\psi_{n_{i}+2} \theta_{i}^{2}+\ldots\right)}\right\}, \mathrm{n}_{i}, \mathrm{i}=1,2, \ldots, \mathrm{k} .
\end{aligned}
$$

Theorem1. The multivariate random vector $\left(X_{1}, X_{2}, \ldots, X_{k}\right)_{\text {having multivariate }}$ power series distribution is uniquely determined by the mean of any one marginal random variable of multivariate random vector $\left(X_{1}, X_{2}, \ldots, X_{k}\right)$.

Proof . Suppose that the mean of the marginal random variable $\boldsymbol{X}_{i}$ is

$$
\begin{aligned}
& m_{i}\left(\theta_{1}, \theta_{2}, \ldots, \theta_{k}\right), \mathrm{i}=1,2, \ldots, \mathrm{k} . \text { Then }, \\
& m_{i}\left(\theta_{1}, \theta_{2}, \ldots, \theta_{k}\right)=\theta_{i} \frac{\partial}{\partial \theta_{i}} \ln f\left(\theta_{1}, \theta_{2}, \ldots, \theta_{k}\right)
\end{aligned}
$$

Let $\mathbf{0}<\boldsymbol{u} \leq \boldsymbol{\theta}_{i} \leq \boldsymbol{t}<\boldsymbol{r}_{i}$. Then for $\boldsymbol{\theta}_{\boldsymbol{i}} \in\left(\mathbf{0}, \boldsymbol{r}_{i}\right)$ we have

$$
\frac{m_{i}\left(\theta_{1}, \theta_{2}, \ldots, \theta_{k}\right)}{\theta_{i}}=\frac{\partial}{\partial \theta_{i}} \ln f\left(\theta_{1}, \theta_{2}, \ldots, \theta_{k}\right)
$$

Therefore, $\int_{u}^{t} \frac{m_{i}\left(\theta_{1}, \theta_{2}, \ldots, \theta_{k}\right)}{\theta_{i}} d \theta_{i}=\frac{\partial}{\partial \theta_{i}} \int_{u}^{t} \ln f\left(\theta_{1}, \theta_{2}, \ldots, \theta_{k}\right) d \theta_{i}$

Implies, $\quad \mathbf{M}_{i}\left(\theta_{1}, \theta_{2}, \ldots, \mathbf{t}, \ldots, \theta_{k}\right)-\mathbf{M}_{i}\left(\theta_{1}, \theta_{2}, \ldots, \mathbf{u}, \ldots, \theta_{k}\right)$

$$
=\ln \left\{\frac{f\left(\theta_{1}, \theta_{2}, \ldots, \mathrm{t}, \ldots, \theta_{k}\right)}{f\left(\theta_{1}, \theta_{2}, \ldots, \mathbf{u}, \ldots, \theta_{k}\right)}\right\}
$$

Where $\frac{\partial}{\partial \theta_{i}} \mathbf{M}_{i}\left(\theta_{1}, \theta_{2}, \ldots, \theta_{k}\right)=m_{i}\left(\theta_{1}, \theta_{2}, \ldots, \theta_{k}\right) / \theta_{i}$

Therefore,

$$
\begin{aligned}
f\left(\theta_{1}, \theta_{2}, \ldots, \mathrm{t}, \ldots, \theta_{k}\right) & =f\left(\theta_{1}, \theta_{2}, \ldots, \mathrm{u}, \ldots, \theta_{k}\right) \mathrm{e}^{-\mathrm{M}_{i}\left(\theta_{1}, \theta_{2}, \ldots, \mathrm{u}, \ldots, \theta_{k}\right)} \cdot \mathrm{e}^{\mathrm{M}_{i}\left(\theta_{1}, \theta_{2}, \ldots, \mathrm{t}, \ldots, \theta_{k}\right)} \\
& =R\left(\theta_{1}, \ldots, \theta_{i-1}, \mathbf{u}, \theta_{i+1}, \ldots, \theta_{k}\right) \mathrm{e}^{\mathrm{M}_{i}\left(\theta_{1}, \theta_{2}, \ldots, \mathrm{t}, \ldots, \theta_{k}\right)}
\end{aligned}
$$

Changing $\mathrm{t}$ by $\theta_{i}$ we get 
Sadoon Abdullah Ibrahim Al-Obaidy et al.

$$
\begin{aligned}
& f\left(\theta_{1}, \theta_{2}, \ldots, \theta_{k}\right)=R\left(\theta_{1}, \ldots, \theta_{i-1}, \mathbf{u}, \theta_{i+1}, \ldots, \theta_{k}\right) \mathrm{e}^{\mathrm{M}_{i}\left(\theta_{1}, \theta_{2}, \ldots, \theta_{k}\right)} \\
& \text { Since } f\left(\theta_{1}, \theta_{2}, \ldots, \theta_{k}\right)=\sum_{x_{1} x_{2} \ldots x_{k}} a_{x_{1} x_{2} \ldots x_{k}} \theta_{1}^{x_{1}} \theta_{2}^{x_{2}} \ldots \theta_{k}^{x_{k}} \\
&=\sum_{x_{i}} \psi_{x_{i}} \theta_{i}^{x_{i}}
\end{aligned}
$$

Therefore, $\mathrm{e}^{\mathbf{M}_{i}\left(\theta_{1}, \theta_{2}, \ldots, \theta_{k}\right)}$ has power series in $\theta_{i}$, say,

$$
\begin{aligned}
\mathrm{P}\left(\mathrm{X}_{i}=x_{i}\right) & =\frac{\mathrm{e}^{\mathrm{M}_{i}\left(\theta_{1}, \theta_{2}, \ldots, \theta_{k}\right)}=\sum_{x_{i}} \psi_{i}^{x_{i}} \theta_{i}^{x_{i}}, \text { Then, }}{f\left(\theta_{1}, \theta_{2}, \ldots, \theta_{k}\right)}=\frac{\psi_{x_{i}} \theta_{i}^{x_{i}}}{R\left(\theta_{1}, \ldots, \mathrm{u}, \ldots, \theta_{k}\right) \mathrm{e}^{\mathrm{M}_{i}\left(\theta_{1}, \ldots, \mathrm{t}, \ldots, \theta_{k}\right)}} \\
& =\frac{\psi_{x_{i}} \theta_{i}^{x_{i}}}{\sum_{x_{i}} \psi_{x_{i}} \theta_{i}^{x_{i}}} \\
& =\frac{\psi_{x_{i}} \theta_{i}^{x_{i}}}{\mathrm{e}^{\mathrm{M}_{i}\left(\theta_{1}, \theta_{2}, \ldots, \theta_{k}\right)}}
\end{aligned}
$$

Hence, without loss of generality we can assume in equation (1) that $\boldsymbol{R}\left(\boldsymbol{\theta}_{1}, \ldots, \mathrm{u}, \ldots, \boldsymbol{\theta}_{\boldsymbol{k}}\right)=\mathbf{1}$ and hence equation (1) becomes

$$
f\left(\theta_{1}, \theta_{2}, \ldots, \theta_{k}\right)=\mathrm{e}^{\mathrm{M}_{i}\left(\theta_{1}, \theta_{2}, \ldots, \theta_{k}\right)}
$$

To prove uniqueness, suppose that $\left(X_{1}, X_{2}, \ldots, X_{k}\right)$ and $\left(Y_{1}, Y_{2}, \ldots, Y_{k}\right)$ are two random vectors having multivariate power series distribution with mean marginal $m_{i}\left(\theta_{1}, \theta_{2}, \ldots, \theta_{k}\right)$ for both. Assume that the corresponding defining functions are $f\left(\theta_{1}, \theta_{2}, \ldots, \theta_{k}\right)$ and $g\left(\theta_{1}, \theta_{2}, \ldots, \theta_{k}\right)$ respectively. Therefore,

$$
\begin{aligned}
\frac{m_{i}\left(\theta_{1}, \theta_{2}, \ldots, \theta_{k}\right)}{\theta_{i}} & =\frac{\partial}{\partial \theta_{i}} \ln f\left(\theta_{1}, \theta_{2}, \ldots, \theta_{k}\right) \\
& =\frac{\partial}{\partial \theta_{i}} \ln g\left(\theta_{1}, \theta_{2}, \ldots, \theta_{k}\right), \text { for all } \theta_{i} \in\left(\mathbf{0 , r _ { i } )}\right.
\end{aligned}
$$

Let $0<\boldsymbol{u} \leq \theta_{i} \leq \boldsymbol{t}<\boldsymbol{r}_{i}$, then,

$$
\int_{u}^{t} \frac{\partial}{\partial \theta_{i}} \ln f\left(\theta_{1}, \theta_{2}, \ldots, \theta_{k}\right) d \theta_{i}=\int_{u}^{t} \frac{\partial}{\partial \theta_{i}} \ln g\left(\theta_{1}, \theta_{2}, \ldots, \theta_{k}\right) d \theta_{i}
$$


Implies

$f\left(\theta_{1}, \theta_{2}, \mathbf{t}, \ldots, \theta_{k}\right)=\mathbf{H}\left(\theta_{1}, \ldots, \theta_{i-1}, \mathbf{u}, \theta_{i+1}, \ldots, \theta_{k}\right) \cdot \mathbf{g}\left(\theta_{1}, \ldots, \mathbf{t}, \ldots, \theta_{k}\right)$

Where $\mathbf{H}\left(\theta_{1}, \ldots, \theta_{i-1}, \mathbf{u}, \theta_{i+1}, \ldots, \theta_{k}\right)=\frac{\mathbf{f}\left(\theta_{1}, \ldots, \mathbf{t}, \ldots, \theta_{k}\right)}{\mathbf{g}\left(\theta_{1}, \ldots, \mathbf{t}, \ldots, \theta_{k}\right)}$

Changing t by $\theta_{i}$ we get

$f\left(\theta_{1}, \theta_{2}, \ldots, \theta_{k}\right)=\mathbf{H}\left(\theta_{1}, \ldots, \theta_{i-1}, \mathbf{u}, \theta_{i+1}, \ldots, \theta_{k}\right) \cdot \mathrm{g}\left(\theta_{1}, \theta_{2}, \ldots, \theta_{k}\right)$

As before and without loss of generality we can assume that:

$\mathbf{H}\left(\theta_{1}, \ldots, \theta_{i-1}, \mathbf{u}, \theta_{i+1}, \ldots, \theta_{k}\right)=\mathbf{1}$ and hence equation (3) becomes

$$
f\left(\theta_{1}, \theta_{2}, \ldots, \theta_{k}\right)=\mathrm{g}\left(\theta_{1}, \theta_{2}, \ldots, \theta_{k}\right), \text { for all } \theta_{i} \in\left(0, r_{i}\right)
$$

Therefore from equation (4) it follows that the two random vectors are identical, which completes the proof of the theorem.

Theorem 2. Let $m_{i}\left(\theta_{1}, \theta_{2}, \ldots, \theta_{k}\right)$ be non-negative and continuous function for all $\theta_{i} \in\left(0, r_{i}\right)$. There exists a multivariate random vector $\left(X_{1}, X_{2}, \ldots, X_{k}\right)$ having $m_{i}\left(\theta_{1}, \theta_{2}, \ldots, \theta_{k}\right)$ as the mean of the marginal random variable $X_{i}$ iff:

(i) There exist a function $M_{i}\left(\theta_{1}, \theta_{2}, \ldots, \theta_{k}\right)$ such that $\frac{\partial}{\partial \theta_{i}} \mathbf{M}_{i}\left(\theta_{1}, \theta_{2}, \ldots, \theta_{k}\right)=m_{i}\left(\theta_{1}, \theta_{2}, \ldots, \theta_{k}\right) / \theta_{i}$, for all $\theta_{i} \in\left(\mathbf{0 , r _ { i }}\right)$,

(ii) $\mathrm{e}^{\mathrm{M}_{i}\left(\theta_{1}, \theta_{2}, \ldots, \theta_{k}\right)}$ has a Maclaurin expansion in $\theta_{1}, \theta_{2}, \ldots, \theta_{k}$ with

Proof. non-negative coefficients.

(A). Assume (i) and (ii) hold. Let

$$
f\left(\theta_{1}, \theta_{2}, \ldots, \theta_{k}\right)=\mathrm{e}^{\mathrm{M}_{i}\left(\theta_{1}, \theta_{2}, \ldots, \theta_{k}\right)}=\sum_{x_{1} x_{2} \ldots x_{k}} a_{x_{1} x_{2} \ldots x_{k}} \theta_{1}^{x_{1}} \theta_{2}^{x_{2}} \ldots \theta_{k}^{x_{k}}
$$

We can define a multivariate random vector $\left(\boldsymbol{X}_{1}, \boldsymbol{X}_{2}, \ldots, \boldsymbol{X}_{k}\right)$ taking values $\left(x_{1}, x_{2}, \ldots, x_{k}\right)$ with probability function

$$
p\left(X_{1}=x_{1}, X_{2}=x_{2}, \ldots, X_{k}=x_{k}\right)=a_{x_{1} x_{2} \ldots x_{k}} \theta_{1}^{x_{1}} \theta_{2}^{x_{2}} \ldots \theta_{k}^{x_{k}} / f\left(\theta_{1}, \theta_{2}, \ldots, \theta_{k}\right)
$$


Therefore, $\left(\boldsymbol{X}_{1}, \boldsymbol{X}_{2}, \ldots, \boldsymbol{X}_{\boldsymbol{k}}\right)$ is a random vector with multivariate power series distribution. Furthermore, the mean of the marginal random variable $\boldsymbol{X}_{i}$ is:

$$
\begin{aligned}
\mu_{i}\left(\theta_{1}, \theta_{2}, \ldots, \theta_{k}\right)=\mathbf{E X} & =\theta_{i} \frac{\partial}{\partial \theta_{i}} \ln f\left(\theta_{1}, \theta_{2}, \ldots, \theta_{k}\right) \\
& =\theta_{i} \frac{\partial}{\partial \theta_{i}} M_{i}\left(\theta_{1}, \theta_{2}, \ldots, \theta_{k}\right) \\
& =m_{i}\left(\theta_{1}, \theta_{2}, \ldots, \theta_{k}\right)
\end{aligned}
$$

(B) Let $\left(X_{1}, X_{2}, \ldots, X_{k}\right)$ be a random vector with a multivariate power series distribution and having defining function $f\left(\theta_{1}, \theta_{2}, \ldots, \theta_{k}\right)$. Then, $m_{i}\left(\theta_{1}, \theta_{2}, \ldots, \theta_{k}\right)=\theta_{i} \frac{\partial}{\partial \theta_{i}} \ln f\left(\theta_{1}, \theta_{2}, \ldots, \theta_{k}\right)$ Implies, $\frac{m_{i}\left(\theta_{1}, \theta_{2}, \ldots, \theta_{k}\right)}{\theta_{i}}=\frac{\partial}{\partial \theta_{i}} \ln f\left(\theta_{1}, \theta_{2}, \ldots, \theta_{k}\right)$, for all $\theta_{i} \in\left(0, r_{i}\right)$. Let $0<u \leq \theta_{i} \leq t<r_{i}$. Therefore,

$$
\int_{u}^{t} \frac{m_{i}\left(\theta_{1}, \theta_{2}, \ldots, \theta_{k}\right)}{\theta_{i}} d \theta_{i}=\int_{u}^{t} \frac{\partial}{\partial \theta_{i}} \ln f\left(\theta_{1}, \theta_{2}, \ldots, \theta_{k}\right) d \theta_{i}
$$

Implies $f\left(\theta_{1}, \ldots, \mathbf{t}, \ldots, \theta_{k}\right)=f\left(\theta_{1}, \ldots, \mathbf{u}, \ldots, \theta_{k}\right) \mathrm{e}^{-\mathrm{M}_{i}\left(\theta_{1}, \ldots, \mathrm{u}, \ldots, \theta_{k}\right)} \cdot \mathrm{e}^{\mathrm{M}_{i}\left(\theta_{1}, \ldots, \mathrm{t}, \ldots, \theta_{k}\right)}$

$$
=K\left(\theta_{1}, \ldots, \theta_{i-1}, \mathbf{u}, \theta_{i+1}, \ldots, \theta_{k}\right) \mathrm{e}^{\mathrm{M}_{i}\left(\theta_{1}, \theta_{2}, \ldots, \mathrm{t}, \ldots, \theta_{k}\right)}
$$

Where $\frac{\partial}{\partial \theta_{i}} \mathbf{M}_{i}\left(\theta_{1}, \theta_{2}, \ldots, \theta_{k}\right)=m_{i}\left(\theta_{1}, \theta_{2}, \ldots, \theta_{k}\right) / \theta_{i}, \theta_{i} \in\left(0, r_{i}\right)$.

Thus (i) is true, changing t by $\boldsymbol{\theta}_{i}$ we get

$$
f\left(\theta_{1}, \theta_{2}, \ldots, \theta_{k}\right)=K\left(\theta_{1}, \ldots, \theta_{i-1}, \mathrm{u}, \theta_{i+1}, \ldots, \theta_{k}\right) \mathrm{e}^{\mathrm{M}_{i}\left(\theta_{1}, \theta_{2}, \ldots, \theta_{k}\right)}
$$

As before and without loss of generality we can assume in equation (5) that $K\left(\theta_{1}, \ldots, \theta_{i-1}, \mathbf{u}, \theta_{i+1}, \ldots, \theta_{k}\right)=\mathbf{1}$, and hence equation (5) becomes

$$
f\left(\theta_{1}, \theta_{2}, \ldots, \theta_{k}\right)=\mathrm{e}^{\mathrm{M}_{i}\left(\theta_{1}, \theta_{2}, \ldots, \theta_{k}\right)}
$$

Since $f\left(\theta_{1}, \theta_{2}, \ldots, \theta_{k}\right)$ has a Maclaurin expansion in $\left(\theta_{1}, \theta_{2}, \ldots, \theta_{k}\right)$ with nonnegative coefficients, then from equation (6) it follows that $\mathbf{e}^{\mathbf{M}_{i}\left(\theta_{1}, \theta_{2}, \ldots, \theta_{k}\right)}$ has a Maclaurin expansion with the same property. Therefore (ii) is true. This completes the proof of the theorem. 


\section{Demonstration Example}

In order to demonstrate the value of these theories, the following example is given. Example: Let

$m_{i}\left(\theta_{1}, \theta_{2}, \ldots, \theta_{k}\right)=-\frac{\theta_{i}}{\left(1-\theta_{1}-\theta_{2}-\ldots-\theta_{k}\right) \ln \left(1-\theta_{1}-\theta_{2}-\ldots-\theta_{k}\right)}$,

$\mathbf{0}<\theta_{i}<\mathbf{1}, \mathbf{i}=\mathbf{1}, 2, \ldots, \mathrm{k}$. Then,

$$
\mathrm{M}_{i}\left(\theta_{1}, \theta_{2}, \ldots, \theta_{k}\right)=\ln \left\{-A \ln \left(1-\theta_{1}-\theta_{2}-\ldots-\theta_{k}\right)\right\}
$$

Where $\mathrm{A}$ is independent of $\theta_{i}$, in general $A=A\left(\theta_{1}, \ldots, \theta_{i-1}, \theta_{i+1}, \ldots, \theta_{k}\right)$

Now $\mathrm{e}^{\mathrm{M}_{i}\left(\theta_{1}, \theta_{2}, \ldots, \theta_{k}\right)}=-\mathrm{A} \ln \left(1-\theta_{1}-\theta_{2}-\ldots-\theta_{k}\right)$

$$
=\mathrm{A} \sum_{x_{1}=1, \ldots x_{k}=1} \frac{\Gamma\left(x_{1}+x_{2}+\ldots+x_{k}\right)}{x_{1} ! x_{2} ! \ldots x_{k} !} \theta_{1}^{x_{1}} \theta_{2}^{x_{2}} \ldots \theta_{k}^{x_{k}}
$$

Therefore, there exists a random vector $\left(X_{1}, X_{2}, \ldots, X_{k}\right)$ having multivariate power series distribution with:

$$
m_{i}\left(\theta_{1}, \theta_{2}, \ldots, \theta_{k}\right)=-\frac{\theta_{i}}{\left(1-\theta_{1}-\theta_{2}-\ldots-\theta_{k}\right) \ln \left(1-\theta_{1}-\theta_{2}-\ldots-\theta_{k}\right)}
$$

as the mean of the marginal random variable $\boldsymbol{X}_{i}$ the defining function of this random vector $\left(X_{1}, X_{2}, \ldots, X_{k}\right)$ is:

$$
f\left(\theta_{1}, \theta_{2}, \ldots, \theta_{k}\right)=-\mathrm{A} \ln \left(1-\theta_{1}-\theta_{2}-\ldots-\theta_{k}\right)
$$

Without loss of generality we can assume $\mathrm{A}=1$, and hence equation (7) becomes

$$
f\left(\theta_{1}, \theta_{2}, \ldots, \theta_{k}\right)=-\ln \left(1-\theta_{1}-\theta_{2}-\ldots-\theta_{k}\right)
$$

Therefore

$p\left(X_{1}=x_{1}, X_{2}=x_{2}, \ldots, X_{k}=x_{k}\right)=-\frac{\Gamma\left(x_{1}+x_{2}+\ldots+x_{k}\right) \theta_{1}^{x_{1}} \theta_{2}^{x_{2}} \ldots \theta_{k}^{x_{k}}}{x_{1} ! x_{2} ! \ldots x_{k} ! \ln \left(1-\theta_{1}-\theta_{2}-\ldots-\theta_{k}\right)}$

This can be called the multivariate logarithmic distribution.

\section{Open Problem and Future Work}

As a future work in this area, the researchers can be focus in the following topics: 
Sadoon Abdullah Ibrahim Al-Obaidy et al.

1- Characterization of polynomial distributions by condition distributions and restricted linear regression.

2- The compound class of general gamma power series distributions.

\section{Conclusion}

In this study the authors has concluded the results that any multivariate power series distribution is determined uniquely from the mean -function of any marginal random variable. Furthermore these results indicated also that any given function satisfying certain conditions construct a random vector with multivariate power series distribution which has a mean of the marginal random variable.

\section{References}

[1] A.Kyriakoussis and H.Papageorgiou, On Characterization of Power Series Distributions by a Marginal Distribution and a Regression Function, Ann. Inst. Statist. Math., 41, No.4 (1989), 671-676.

[2] B.R.Rao and K.G.Janardan , On the Moments of Multivariate Discrete Distributions Using Finite Difference Operators, The American Statistician , 36 (1982),381-383.

[3] C.G.Khatri, On Certain Properties of Power Series Distributions, Biometrika, 46,No.3/4(1959),486-490.

[4] C.G.Khatri, Characterization of Some Discrete Distributions By Linear Regression, J.Indian Statist. Assoc., 16(1978a), 49-58.

[5] C.G.Khatri, Characterization of Some Multivariate Distributions By Conditional Distributions and Linear Regression,J. Indian Statist . ASSOC., 16(1978b), 59-70.

[6] E.Mahmoudi and A.A.Jafari, Generalized Exponential - Power Series Distributions, Computational Statistics and Data Analysis, 56(2012), 4047-4066.

[7] F.Momeni, The Generalized Power Series Distribution and Their Application, The Journal of Mathematics and Computer Science , 2 ,No.4 (2011), 691697 .

[8] H.Papageorgiou, On Characterizing Some Bivariate Discrete Distributions , Austral .J. Statist ., 25(1983), 136-144.

[9] H.Papageorgiou , On Characterizing Some Discrete Distributions By a Conditional Distribution and Regression Funtion, Biometrical,J., 27(1985),473-479.

[10] J.K. Ghosh , K.S.Bikas and K.S.Bimal ,Multivariate Power Series Distributions and Neyman's Properties For Multinomials, Journal of Multivariate Analysis , 7 (1977),397-408. 
[11] J.Wesolowski , Bivariate Discrete Measures Via a Power Series Conditional Distribution and Regression, Journal Of Multivariate Analysis,55(1995),219229.

[12] M.K.Patil and D.T.Shirke, Testing Parameter of the Power Series Distribution of a zero-Inflated Power Series Model ,Statistical Methodology , 4,No.4(2007),393-406.

[13] M.K.Patil and D.T.Shirke, Test for Equality of Inflated parameter of Two Zero-Inflated Power Series Distribution, Communications in Statistics, 40, No.14 (2011), 2539-2553.

[14] N.L.Johnson, S.Kotz and A.W.Kemp , Univariate Discrete Distributions , Wiley, New York (1992).

[15] R.B.Silva , M.Bourguignon ,C.R.B. Dias and G.M.Cordeiro, The Compound Class of Extended Weibull Power Series Distributions, Computational Statistics and Data Analysis ,58(2013),352-367.

[16] R.C.Dahiya and R.M.Korwar, On Characterizing Some Bivariate Discrete Distributions By Linear Regression, Sankhya:The Indian Journal of Statistics, 39(1977), 124-129.

[17] R.Gupta and K.K.Das, A Family of Multivariate Abel Series Distributions of Order K, Applied Mathematical Sciences, 2, No.45 (2008), 2239-2246.

[18] R.M.Korwar ,ON Characterizing Some Discrete Distributions By Linear Regression, Comm. Statist.,4(1975),1133-1147.

[19] S.R.Patel and V.Vidyanagar, Minimum Variance Unbiased Estimation of Left Truncated Multivariate Modified Power Series Distribution, Metrika, 26(1979), 87-94.

[20] S.Simic, On Moments of the Power Series Distributions, The Open Statistics and Probability Journal, 1(2009), 3-6. 